\title{
Medication Adherence Trajectories: A Systematic Literature Review
}

\author{
Mai Alhazami, BPharm, MSc, PhD; Vasco M. Pontinha, MPharm, MA; \\ Julie A. Patterson, PharmD, PhD; and David A. Holdford, PhD
}

\begin{abstract}
BACKGROUND: Traditional adherence measures such as proportion of days covered (PDC) and medication possession ratio (MPR) are limited in their ability to explain patient medication adherence over time. Group-based trajectory modeling (GBTM) is a new methodological approach that visually describes the dynamics of long-term medication adherence and classifies adherence behavior into groups.

OBJECTIVES: To describe and compare trajectories of medication nonadherence reported in the medical literature, including identifying consistent trends in adherence trajectories and disease and patient characteristics that predict trajectory group membership.

METHODS: A systematic literature review was conducted in April 2020 in PubMed and CINAHL using MeSH terms and key words in appropriate combinations. Citations were screened for relevance using predefined inclusion and exclusion criteria and evaluated according to variables associated with group-based trajectory models.
\end{abstract}

RESULTS: 21 articles met the study criteria and were reviewed. Generally, studies identified 4 to 6 trajectory groups that described longitudinal medication adherence behavior. Most commonly identified trajectories were labeled as (a) consistent, high adherence, (b) declining adherence, (c) early and consistent nonadherence, and (d) initial nonadherence followed by an increase. Several predictors, including socioeconomic status, disease characteristics, and therapy initiation were routinely associated with group membership.

CONCLUSIONS: This review suggests that adherence trajectories and predictors of specific group membership may be similar across diverse disease states. GBTM describes longitudinal, dynamic patterns of medication adherence that may facilitate the development of targeted interventions to promote adherence. Implications for value-based payment systems are discussed in this review.

J Manag Care Spec Pharm. 2020;26(9):1138-52

Copyright $\odot 2020$, Academy of Managed Care Pharmacy. All rights reserved.

\section{What is already known about this subject}

Static measures such as proportion of days covered or medication possession ratio may not adequately capture the fluid nature of medication-taking behavior and allow the identification of underlying factors that predict nonadherence behavior over a long period of time (e.g., over a period of 1 year).

Group-based trajectory modeling (GBTM), which describes different medication adherence patterns as trajectories over time, has been increasingly used to investigate medication adherence patterns.

\section{What this study adds}

GBTM on oral medication adherence consistently identifies 4 to 6 similar medication adherence behavior trajectories irrespective of the disease state.

Similar demographic and clinical characteristics have been shown to predict trajectory group membership across diverse disease states.

This review concludes that GBTM may be a more appropriate approach to investigating and designing practice interventions for improving medication adherence.

$\mathrm{M}$ edication nonadherence is a major obstacle to improving health care outcomes in long-term therapies for chronic diseases. ${ }^{1}$ It is associated with adverse health outcomes and higher health care costs. ${ }^{1-3}$ Studies on medication adherence commonly use administrative claims data to quantify adherence with established metrics such as proportion of days covered (PDC) and medication possession ratio (MPR). ${ }^{4-6}$ These measures, which differ subtly in their calculations, summarize overall adherence with a single number based on the number of days a patient has medication on hand during a specified time period and assume that medication on hand is taken.

While PDC and MPR are well established in the literature and have garnered endorsements from organizations such as the Pharmacy Quality Alliance and the National Quality Forum, ${ }^{7,8}$ their limitations are also well documented. ${ }^{4,9}$ PDC and MPR measures can be insensitive to changes in patient behavior over time and circumstances. ${ }^{10,11}$ They are also most often used to classify patients dichotomously, with ratios above a specified threshold (e.g., PDC $>80 \%$ ) denoting adherence, ${ }^{10,11}$ which may not adequately differentiate between clinically significant adherence behaviors. For example, a PDC of $79 \%$ is labeled nonadherent and a PDC of $81 \%$ is considered adherent, although the probability of therapeutic success is likely to be similar.

In reality, adherence to medication therapy is a dynamic process consisting of a series of stages of adherence and nonadherence over time during initiation, maintenance, and discontinuation phases of treatment. ${ }^{12}$ This dynamic process is influenced by multiple factors related to socioeconomics, the health care system, diseases, treatments or regimens, and patients. ${ }^{1,13}$ 
The fluid nature of medication-taking behavior and the underlying factors driving that behavior are not adequately captured with PDC and MPR ratios alone. Patient adherence may vary over time depending on an individual's employment status, family situation, new disease diagnosis, change in therapy, or any host of other factors. By quantifying adherence with a single ratio, PDC and MPR collapse a broad spectrum of adherence behaviors into a single number, thereby masking complex, dynamic, and longitudinal patterns of behavior. ${ }^{10,14}$ Indeed, a patient with a PDC of 60\% may have been (a) highly adherent during the early stages of therapy but less adherent as time went on, (b) poorly adherent during the early stages of therapy but more adherent over time, or (c) intermittently adherent throughout the follow-up period. ${ }^{10}$ Each of these 3 patterns of taking medication would be classified as nonadherent using conventional measures and would be treated as a homogenous adherence group. Ignoring these underlying differences in the refill patterns and patients' adherence behavior may lead to missed opportunities to address barriers to appropriate medication use.

Group-based trajectory modeling (GBTM) is a method commonly used in the social sciences that elicits common trajectories, or progressions, of how a group of people behaves over a period of time. Lately, it has been used to evaluate adherence, overcoming the limitations of static measures such as annual PDC or MPR. Instead of providing a single adherence ratio, it describes different medication adherence patterns as trajectories over time.

With GBTM, patient administrative claims data are used to calculate monthly measures of adherence, which are then analyzed over time, clustering patients into similar patterns of longitudinal adherence behavior (e.g., always adherent or decreasingly adherent). ${ }^{10}$ The number of trajectory groups may be identified based on several criteria, including Bayesian information criteria, where a lower value indicates better fit, ${ }^{15}$ and Nagin's criteria for model adequacy, ${ }^{16}$ with consideration given to group size such that the proportion of patients in each group is not less than 5\% of the total sample. . $^{1517}$ Each method follows specific criteria to classify adherence groups based on their membership probabilities (highest likelihood probability to belong to certain groups). Once the final number of groups is selected, trajectories are plotted and presented in graphical form. Regression models may then be used to estimate and characterize the predictors of each trajectory. ${ }^{10,17}$

Given the growing literature on GBTM medication adherence studies, there is a need to examine the commonalities and differences in models and the trajectories of adherence identified by them. If pharmacists, especially those within managed care organizations, can identify common different adherence trajectories and their underlying causes, they can develop population-based interventions to better address nonadherence. This systematic literature review describes studies that used group-based trajectory models to identify common patterns of medication adherence. The review summarizes general characteristics of the studies concerning therapies chosen, populations examined, and study features.

\section{Methods}

\section{Search Strategy}

The literature review was conducted on April 2020 in PubMed and CINAHL databases using MeSH terms and key words in appropriate combinations and in accordance with PRISMA guidelines. Search terms were combinations of the following: (group based trajectory modeling OR group based trajectory models OR group based trajectory OR trajectory) AND (medication adherence OR "Medication Adherence"[Mesh]), (group based trajectory modeling OR group based trajectory models OR group based trajectory OR trajectory) AND (fill prescription), (group based trajectory modeling OR group based trajectory models OR group based trajectory OR trajectory) AND (medication compliance), (group based trajectory modeling OR group based trajectory models OR group based trajectory OR trajectory) AND (medication persistence). To ensure complete capture of relevant articles, the authors conducted backward snowballing, by reviewing the references of the included articles.

\section{Review Procedures and Study Selection}

Titles and abstracts from search result articles were screened by 2 investigators based on inclusion and exclusion criteria. Disagreements pertaining to the inclusion and exclusion criteria were resolved by discussion with a third researcher. Studies that evaluated patient adherence to oral medications over time using group-based trajectory models and were published in English were included. Studies focusing on select special populations (i.e., children and pregnant women) were excluded because of the unique nature of medication-taking behaviors in these groups. Studies examining adherence to provider-administered biologics were excluded because adherence behavior may systematically differ from self-administered medications obtained from a pharmacy. Review articles, case reports, and prospective studies without results were also excluded.

\section{Data Extraction}

Two researchers extracted data regarding authorship, year of publication, country, population, type of treatment, sample size, number and shape of trajectory groups identified, clinical outcomes that were being assessed, and general conclusions that results from GBTM analysis.

\section{Results}

The search in PubMed and CINAHL yielded 248 articles. After screening for duplicates and applying inclusion and exclusion criteria, 20 research articles remained for analysis (Figure 1). 


\section{FIGURE 1 PRISMA Flowchart}

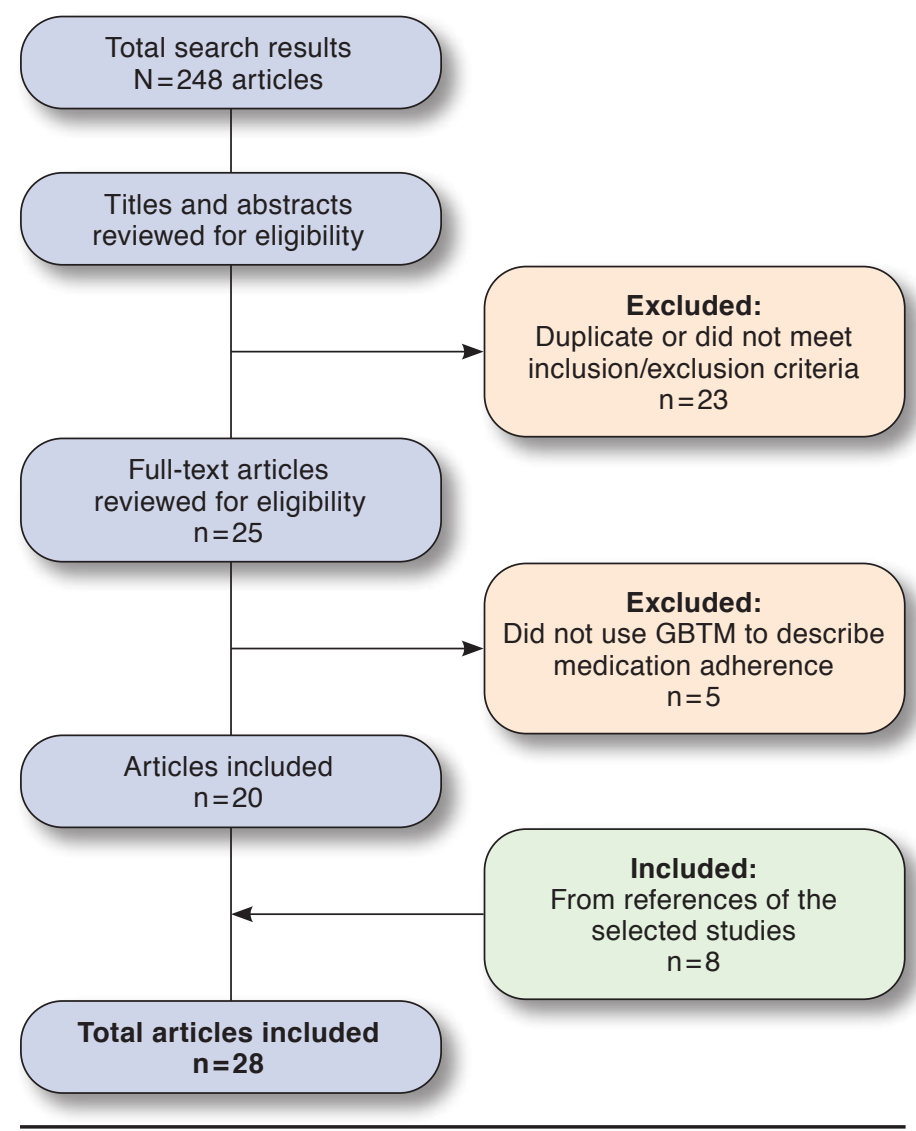

GBTM = group-based trajectory modeling. PRISMA = Preferred Reporting Items for Systematic Reviews and Meta-Analyses.

After the full-text review, reference lists for included articles were searched, yielding 8 additional studies for inclusion.

All 28 papers were published in the last 10 years (20102020). Most studies were conducted in the United States $(\mathrm{n}=17,60.7 \%),{ }^{10,18-34}$ while the remaining were conducted in Europe $(n=7,25 \%),{ }^{35-41}$ Taiwan $(n=1,3.6 \%),{ }^{42}$ and Australia $(\mathrm{n}=2,7.14 \%){ }^{43,44}$ Although a variety of medical conditions were explored in GBTM populations, the majority of studies focused on populations diagnosed with cardiovascular diseases ( $n=19$, $67.9 \%) .10,18,20,22-24,27,29,30,32-38,41,43,45$ Study duration ranged from 9 months to 6 years, ${ }^{38,42}$ with most lasting 2 years or less $(\mathrm{n}=22,78.6 \%)$. Table 1 summarizes the studies included in this review by the population characteristics, type of treatment, study duration, adherence measure, country, sample size, number of trajectory groups identified, and overview of the conclusions.

\section{Number and Types of Trajectory Groups}

In Table 1, the column "Trajectory Group" encompasses the number and general description of the adherence trajectories found in each of the included studies. The number of trajectory groups identified ranged from 3 to 7 , although most studies ( $n=18,85.7 \%$ ) identified 4 to 6 trajectory groups. Regardless of the total number of trajectories identified, 19 studies identified the following 4 similar adherence trajectory groups: (1) consistent, high adherence, (2) declining adherence, (3) early and consistent nonadherence, and (4) initial nonadherence followed by a slight increase in adherence.

Consistent, High Adherence. All of the published studies identified a "perfect adherence" or "nearly perfect adherence" trajectory composed of patients who consistently registered a monthly probability of PDC or MPR over $80 \%$ for the duration of the study. In most studies, ( $n=22,78.6 \%)$, the consistent, high adherence trajectory group comprised the largest proportion of patients, ranging from $21 \%$ to $80 \%$ of patients in the study. More specifically, 14 studies observed a consistently adherent group that represented more than $40 \%$ of the sample. ${ }^{18-20,22-26,29,32,33,35-38,42-45}$

Declining Adherence. Studies that identified more than 1 declining adherence trajectory varied primarily in the shape and speed of decline. The most commonly identified declining adherence trajectory $(n=26,92.9 \%)$ was one characterized by a gradual decline in adherence, with "falling" adherence rates starting anywhere between the third and ninth month. ${ }^{10,19,20,22-24,26-28,30,37-39,42,43,46}$ Studies also often identified a rapid trajectory $(n=22,78.6 \%)$, in which adherence immediately declines within the first 2 months of therapy initiation. $10,19,20,22-24,26-28,30,33,37,39,40,43-46$

Early and Consistent Nonadherence. All articles reported early nonadherent behaviors. In these trajectory groups, adherence steeply declined well below $80 \%$ after the first months. In some studies $(n=4,14.3 \%)$, this group was designated as rapid decline in adherence, or persistent nonadherers. ${ }^{20,23,43,46}$ In all studies, the steep decline in adherence was usually followed by a consistently very low adherence throughout the study duration. However, adherence analyses based on administrative claims data require at least 1 fill of the prescribed medicine. Notably, adherence measures using administrative claims data do not capture patients with primary nonadherence who never pick up their initial fill. Group-based trajectory models using claims data can only identify patients with secondary nonadherence and may therefore underestimate the true proportion of consistently nonadherent patients, which would more accurately include patients with primary and secondary nonadherence.

Initial Nonadherence Followed by an Increase. The fourth commonly identified trajectory group described patients whose 


\section{TABLE 1 Summary of Identified Studies}

\begin{tabular}{|c|c|c|c|c|c|c|c|c|c|c|}
\hline Authors & Objectives & Population & $\begin{array}{c}\text { Type of } \\
\text { Treatment }\end{array}$ & $\begin{array}{l}\text { Length } \\
\text { of Study } \\
\text { Period }\end{array}$ & Country & $\begin{array}{c}\text { Sample } \\
\text { Size }\end{array}$ & $\begin{array}{c}\text { Adherence } \\
\text { Measure }\end{array}$ & $\begin{array}{l}\text { Trajectory } \\
\text { Group }\end{array}$ & $\begin{array}{l}\text { Clinical } \\
\text { Outcomes }\end{array}$ & Conclusions \\
\hline $\begin{array}{l}\text { Dillon } \\
\text { et al., } \\
2019^{36}\end{array}$ & \begin{tabular}{|l} 
1. Classify adherence \\
to antihypertensives \\
using PDC and GBTM \\
2. Evaluate the longi- \\
tudinal association \\
between community \\
pharmacy antihyper- \\
tensive refill adher- \\
ence metrics (GBTM \\
and PDC) and the \\
number of hospital \\
visits and GP visits
\end{tabular} & $\begin{array}{l}\text { Patients aged } \\
\geq 65 \text { years }\end{array}$ & \begin{tabular}{|l|} 
Multiple \\
classes of \\
antihyper- \\
tensives
\end{tabular} & $\begin{array}{c}1 \\
\text { year }\end{array}$ & $\begin{array}{l}\text { Republic } \\
\text { of Ireland }\end{array}$ & 905 & PDC & $\begin{array}{l}\text { 1. Perfect } \\
\text { adherence } \\
\text { 2. High } \\
\text { adherence } \\
\text { 3. Low } \\
\text { adherence }\end{array}$ & $\begin{array}{l}\text { Hospital visit, } \\
\text { GP visit }\end{array}$ & $\begin{array}{l}\text { PDC could } \\
\text { be used to } \\
\text { rank patients } \\
\text { by adherence } \\
\text { level and be } \\
\text { combined with } \\
\text { other adher- } \\
\text { ence measure- } \\
\text { ments, such } \\
\text { as individual } \\
\text { adherence tra- } \\
\text { jectory graphs, } \\
\text { to provide a } \\
\text { richer picture of } \\
\text { patient adher- } \\
\text { ence behavior }\end{array}$ \\
\hline $\begin{array}{l}\text { Marcum } \\
\text { et al., } \\
201918\end{array}$ & $\begin{array}{l}\text { 1. Examine antihyper- } \\
\text { tensive and statin } \\
\text { adherence } \\
\text { 2. Compare people who } \\
\text { went on to develop } \\
\text { dementia with those } \\
\text { who did not }\end{array}$ & $\begin{array}{l}\text { Patients aged } \\
\geq 65 \text { years }\end{array}$ & \begin{tabular}{|l|} 
Multiple \\
classes of \\
antihyper- \\
tensives \\
and statins
\end{tabular} & 3 years & $\begin{array}{l}\text { United } \\
\text { States }\end{array}$ & 4,368 & PDC & \begin{tabular}{|l|} 
1. Perfect \\
adherence \\
2. Moderate \\
nonadher- \\
ence \\
3. Slow decline \\
4. Rapid \\
decline
\end{tabular} & - & $\begin{array}{l}\text { Patterns of } \\
\text { medication } \\
\text { adherence may } \\
\text { be useful to } \\
\text { identify a sub- } \\
\text { set of people at } \\
\text { higher likeli- } \\
\text { hood of devel- } \\
\text { oping dementia }\end{array}$ \\
\hline $\begin{array}{l}\text { Winn } \\
\text { et al., } \\
201919\end{array}$ & $\begin{array}{l}\text { 1. Examine how adher- } \\
\text { ence to ET for women } \\
\text { with breast cancer } \\
\text { was impacted by } \\
\text { reducing copayments } \\
\text { for ETs by the intro- } \\
\text { duction of generic } \\
\text { ETs among women } \\
\text { who do not receive } \\
\text { a subsidy compared } \\
\text { with those that do } \\
\text { receive a subsidy } \\
\text { and are not exposed } \\
\text { to any changes in } \\
\text { copayments by using } \\
\text { GBTM }\end{array}$ & $\begin{array}{l}\text { Women with } \\
\text { breast cancer } \\
\text { aged }>66 \\
\text { years }\end{array}$ & ET & 1 year & $\begin{array}{l}\text { United } \\
\text { States }\end{array}$ & 3,344 & PDC & \begin{tabular}{|l} 
1. Perfect \\
adherence \\
2. Slow \\
decline then \\
increase \\
3. Rapid \\
decline, \\
then \\
increase \\
4. Slow decline \\
5. Rapid \\
decline \\
6. Low adher- \\
ence
\end{tabular} & - & $\begin{array}{l}\text { Describes a } \\
\text { new approach } \\
\text { to identify } \\
\text { heterogenous } \\
\text { effects when } \\
\text { using an } \\
\text { interrupted } \\
\text { time series } \\
\text { research design }\end{array}$ \\
\hline $\begin{array}{l}\text { MacEwan } \\
\text { et al., } \\
2018^{20}\end{array}$ & $\begin{array}{l}\text { 1. Identify patients' } \\
\text { antipsychotic } \\
\text { adherence patterns } \\
\text { 2. Assess whether } \\
\text { antipsychotic } \\
\text { adherence patterns } \\
\text { can predict other } \\
\text { medications } \\
\text { adherence patterns }\end{array}$ & $\begin{array}{l}\text { Patients with } \\
\text { serious men- } \\
\text { tal illness } \\
\text { who initiated } \\
\text { an atypical } \\
\text { antipsychotic } \\
\text { and were also } \\
\text { taking an } \\
\text { SSRI, bigu- } \\
\text { anide, or an } \\
\text { ACE inhibitor }\end{array}$ & \begin{tabular}{|l|} 
Atypical \\
antipsy- \\
chotic, \\
SSRI, \\
biguanides, \\
and ACE \\
inhibitors
\end{tabular} & 1 year & $\begin{array}{l}\text { United } \\
\text { States }\end{array}$ & 431,591 & PDC & 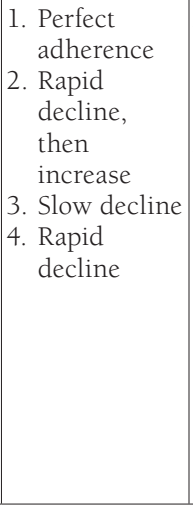 & $\begin{array}{l}\text { Adherence } \\
\text { patterns for } \\
\text { SSRIs, bigu- } \\
\text { anides, and } \\
\text { ACE inhibitors }\end{array}$ & $\begin{array}{l}\text { Among patients } \\
\text { with multiple } \\
\text { chronic mental } \\
\text { and physical } \\
\text { illnesses, pat- } \\
\text { terns of atypical } \\
\text { antipsychotic } \\
\text { adherence were } \\
\text { useful predic- } \\
\text { tors of adher- } \\
\text { ence patterns } \\
\text { to a patient's } \\
\text { adherence to } \\
\text { ACE inhibitors, } \\
\text { biguanides, and } \\
\text { SSRIs }\end{array}$ \\
\hline
\end{tabular}




\section{TABLE 1 Summary of Identified Studies (continued)}

\begin{tabular}{|c|c|c|c|c|c|c|c|c|c|c|}
\hline Authors & Objectives & Population & $\begin{array}{c}\text { Type of } \\
\text { Treatment }\end{array}$ & $\begin{array}{l}\text { Length } \\
\text { of Study } \\
\text { Period }\end{array}$ & Country & $\begin{array}{c}\text { Sample } \\
\text { Size }\end{array}$ & $\begin{array}{l}\text { Adherence } \\
\text { Measure }\end{array}$ & $\begin{array}{l}\text { Trajectory } \\
\text { Group }\end{array}$ & $\begin{array}{l}\text { Clinical } \\
\text { Outcomes }\end{array}$ & Conclusions \\
\hline $\begin{array}{l}\text { Feldman } \\
\text { et al., } \\
2018^{46}\end{array}$ & $\begin{array}{l}\text { 1. Identify adherence } \\
\text { trajectories for } \\
\text { patients taking HCQ } \\
\text { 2. Identify predictors } \\
\text { of nonadherence to } \\
\text { HCQ }\end{array}$ & \begin{tabular}{|l|} 
Adult \\
Medicaid \\
patients with \\
systemic \\
lupus \\
erythema- \\
tosus
\end{tabular} & HCQ & 1 year & $\begin{array}{l}\text { United } \\
\text { States }\end{array}$ & 10,406 & PDC & $\begin{array}{l}\text { 1. } \text { Perfect } \\
\text { adherence } \\
\text { 2. Moderate } \\
\text { nonadher- } \\
\text { ence } \\
\text { 3. Low decline } \\
\text { 4. Very rapid } \\
\text { decline }\end{array}$ & - & $\begin{array}{l}\text { HCQ adherence } \\
\text { is a dynamic } \\
\text { behavior that } \\
\text { declines over } \\
\text { the first year } \\
\text { of use }\end{array}$ \\
\hline $\begin{array}{l}\text { Franklin } \\
\text { et al., } \\
2018^{22}\end{array}$ & $\begin{array}{l}\text { 1. Identify adherence } \\
\text { trajectories for statins, } \\
\text { antihypertensives, } \\
\text { and oral antidiabetics } \\
\text { 2. Identify predictors of } \\
\text { nonadherence } \\
\text { trajectories } \\
\text { 3. Evaluate the accuracy } \\
\text { of predictions of } \\
\text { medication adherence } \\
\text { based on EHR data } \\
\text { versus claims }\end{array}$ & \begin{tabular}{|l|} 
Tufts Health \\
Plan Medicare \\
Advantage \\
beneficiaries \\
aged 65 and \\
older, receiv- \\
ing care at \\
Harvard \\
Vanguard \\
Medical \\
Associates
\end{tabular} & \begin{tabular}{|l|} 
Statins, \\
multiple \\
classes of \\
antihyper- \\
tensives, \\
and oral \\
antidiabetics
\end{tabular} & 1 year & $\begin{array}{l}\text { United } \\
\text { States }\end{array}$ & 11,479 & PDC & \begin{tabular}{|l} 
1. Perfect \\
adherence \\
2. Decline after \\
9 months \\
3. Moderate \\
nonadher- \\
ence \\
4. Decline after \\
6 months \\
5. Rapid \\
decline
\end{tabular} & - & $\begin{array}{l}\text { EHR data can } \\
\text { provide good } \\
\text { predictions of } \\
\text { adherence } \\
\text { trajectory }\end{array}$ \\
\hline $\begin{array}{l}\text { Schaffer } \\
\text { et al., } \\
2017^{43}\end{array}$ & $\begin{array}{l}\text { 1. Compare statin } \\
\text { adherence in } \\
\text { individuals } \\
\text { initiating combined } \\
\text { amlodipine/ } \\
\text { atorvastatin therapy } \\
\text { as FDC or free } \\
\text { combination } \\
\text { 2. Identified subgroups } \\
\text { benefiting most from } \\
\text { FDCs } \\
\text { 3. Identified predictors } \\
\text { of adherence } \\
\text { trajectories }\end{array}$ & $\begin{array}{l}\text { All patients } \\
\text { initiating } \\
\text { amlodipine in } \\
\text { combination } \\
\text { with atorvas- } \\
\text { tatin, either } \\
\text { as FDC or in } \\
\text { free combina- } \\
\text { tion }\end{array}$ & $\begin{array}{l}\text { Amlodipine } \\
\text { and atorv- } \\
\text { astatin as } \\
\text { FDC and } \\
\text { free } \\
\text { combina- } \\
\text { tion }\end{array}$ & 2 years & Australia & $\begin{array}{l}3,996 \\
(\text { FDC), } \\
5,434 \\
\text { (free } \\
\text { combi- } \\
\text { nation) }\end{array}$ & PDC & \begin{tabular}{|l|} 
1. Perfect \\
adherence \\
2. Moderate \\
adherence \\
3. Slow decline \\
4. Very rapid \\
decline
\end{tabular} & - & $\begin{array}{l}\text { The amlodip- } \\
\text { ine/atorvas- } \\
\text { tatin FDC was } \\
\text { associated with } \\
\text { greater statin } \\
\text { adherence } \\
\text { among preva- } \\
\text { lent statin users }\end{array}$ \\
\hline $\begin{array}{l}\text { Dillon } \\
\text { et al., } \\
2018^{35}\end{array}$ & $\begin{array}{l}\text { 1. Characterize adher- } \\
\text { ence to antihyperten- } \\
\text { sive medication } \\
\text { 2. Test predictive valid- } \\
\text { ity of GBTM in BP } \\
\text { measurement }\end{array}$ & $\begin{array}{l}\text { Patients aged } \\
\geq 65 \text { years }\end{array}$ & \begin{tabular}{|l|} 
Multiple \\
classes of \\
antihyper- \\
tensives \\
and statins
\end{tabular} & 1 year & $\begin{array}{c}\text { Republic of } \\
\text { Ireland }\end{array}$ & 905 & PDC & $\begin{array}{l}\text { 1. Perfect } \\
\text { adherence } \\
\text { 2. High adher- } \\
\text { ence } \\
\text { 3. Moderate } \\
\text { nonadher- } \\
\text { ence }\end{array}$ & - & $\begin{array}{l}\text { GBTM identi- } \\
\text { fied } 3 \text { trajecto- } \\
\text { ries; however, } \\
\text { did not show } \\
\text { predictive valid- } \\
\text { ity with BP } \\
\text { measurement } \\
\end{array}$ \\
\hline $\begin{array}{l}\text { Hargrove } \\
\text { et al., } \\
2017^{23}\end{array}$ & $\begin{array}{l}\text { 1. Use GBTM to iden- } \\
\text { tify antihypertensive } \\
\text { adherence trajectories } \\
\text { 2. Comapre adherence } \\
\text { trajectories to tra- } \\
\text { ditional adherence } \\
\text { measures } \\
\text { 3. Identify patients } \\
\text { characteristics associ- } \\
\text { ated with adherence } \\
\text { trajectories }\end{array}$ & $\begin{array}{l}\text { Medicare } \\
\text { patients aged } \\
\geq 65 \text { years }\end{array}$ & \begin{tabular}{|l|} 
Multiple \\
classes of \\
antihyper- \\
tensives \\
and statins
\end{tabular} & 1 year & $\begin{array}{l}\text { United } \\
\text { States }\end{array}$ & 282,520 & $\begin{array}{l}\text { PDC: } \text { used } \\
\text { to compare } \\
\text { identifying } \\
\text { adherent } \\
\text { and non- } \\
\text { adherent } \\
\text { months }\end{array}$ & $\begin{array}{l}\text { 1. Perfect } \\
\text { adherence } \\
\text { 2. Rapid } \\
\text { decline, } \\
\text { then } \\
\text { increase } \\
\text { 3. Moderate } \\
\text { decline, } \\
\text { then } \\
\text { increase } \\
\text { 4. Moderate } \\
\text { decline } \\
\text { 5. Rapid } \\
\text { decline } \\
\text { 6. Very rapid } \\
\text { decline }\end{array}$ & - & $\begin{array}{l}\text { GBTM is } \\
\text { an effective } \\
\text { method to } \\
\text { identify } \\
\text { patterns of } \\
\text { medication } \\
\text { adherence } \\
\text { compared with } \\
\text { PDC }\end{array}$ \\
\hline
\end{tabular}




\section{TABLE 1 Summary of Identified Studies (continued)}

\begin{tabular}{|c|c|c|c|c|c|c|c|c|c|c|}
\hline Authors & Objectives & Population & $\begin{array}{c}\text { Type of } \\
\text { Treatment }\end{array}$ & $\begin{array}{l}\text { Length } \\
\text { of Study } \\
\text { Period }\end{array}$ & Country & $\begin{array}{c}\text { Sample } \\
\text { Size }\end{array}$ & $\begin{array}{c}\text { Adherence } \\
\text { Measure }\end{array}$ & $\begin{array}{l}\text { Trajectory } \\
\text { Group }\end{array}$ & $\begin{array}{l}\text { Clinical } \\
\text { Outcomes }\end{array}$ & Conclusions \\
\hline $\begin{array}{l}\text { MacEwan } \\
\text { et al., } \\
2016^{24}\end{array}$ & $\begin{array}{l}\text { 1. Identify adherence } \\
\text { trajectory for } \\
\text { oral atypical } \\
\text { antipsychotics } \\
\text { 2. Identify associated } \\
\text { factors }\end{array}$ & \begin{tabular}{|l|} 
Adult \\
schizophrenia \\
patients
\end{tabular} & $\begin{array}{l}\text { Oral } \\
\text { atypical } \\
\text { antipsy- } \\
\text { chotic }\end{array}$ & 1 year & $\begin{array}{l}\text { United } \\
\text { States }\end{array}$ & 29,607 & PDC & \begin{tabular}{|l} 
1. Perfect \\
adherence \\
2. Decline after \\
3 months \\
3. Decline after \\
6 months \\
4. Decline after \\
9 months \\
5. Rapid \\
decline, \\
then \\
increase \\
after 6 \\
months \\
6. Very rapid \\
decline
\end{tabular} & $\begin{array}{l}\text { Psychiatric } \\
\text { inpatient } \\
\text { admission } \\
\text { and ED visit }\end{array}$ & $\begin{array}{l}\text { Adherence } \\
\text { patterns } \\
\text { identified by } \\
\text { GBTM are } \\
\text { more varied } \\
\text { than research } \\
\text { based on PDC; } \\
\text { lower adherence } \\
\text { trajectories } \\
\text { associated with } \\
\text { higher ED visits }\end{array}$ \\
\hline $\begin{array}{l}\text { Aarnio } \\
\text { et al., } \\
2016^{37}\end{array}$ & $\begin{array}{l}\text { 1. Identify adherence } \\
\text { trajectories of statin } \\
\text { 2. Examine association } \\
\text { between SEP and } \\
\text { adherence trajectories }\end{array}$ & \begin{tabular}{|l|} 
Patients aged \\
$45-75$ years
\end{tabular} & Statin & $\begin{array}{c}18 \\
\text { months }\end{array}$ & Finland & 116,846 & $\begin{array}{l}\text { PDC: used } \\
\text { to compare } \\
\text { predictors } \\
\text { ability to } \\
\text { identify } \\
\text { nonadher- } \\
\text { ent partici- } \\
\text { pants }\end{array}$ & \begin{tabular}{|l|} 
1. Perfect \\
adherence \\
2. High adher- \\
ence \\
3. Rapid \\
decline, \\
then \\
increase \\
4. Moderate \\
decline \\
5. Rapid \\
decline \\
6. Very rapid \\
decline
\end{tabular} & - & $\begin{array}{l}\text { SEP is } \\
\text { associated with } \\
\text { low adherence } \\
\text { groups; overall, } \\
\text { GBTM provides } \\
\text { insight to } \\
\text { dynamics of } \\
\text { adherence } \\
\text { behavior }\end{array}$ \\
\hline $\begin{array}{l}\text { Librero } \\
\text { et al., } \\
2016^{38}\end{array}$ & $\begin{array}{l}\text { 1. Identify adherence } \\
\text { trajectories for ACEI, } \\
\text { statin, beta blocker, } \\
\text { and antiplatelet } \\
\text { 2. Identify associated } \\
\text { factors }\end{array}$ & $\begin{array}{l}\text { Patients dis- } \\
\text { charged with } \\
\text { CHD }\end{array}$ & $\begin{array}{l}\text { ACEI/ARB, } \\
\text { beta block- } \\
\text { ers, statins, } \\
\text { and anti- } \\
\text { platelet }\end{array}$ & $\begin{array}{c}9 \\
\text { months }\end{array}$ & Spain & 7,462 & PDC & 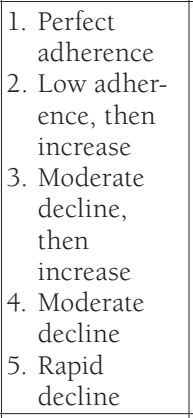 & - & $\begin{array}{l}\text { GBTM } \\
\text { identified } \\
\text { distinct } \\
\text { adherence } \\
\text { trajectories } \\
\text { for differences } \\
\text { in preventive } \\
\text { medication for } \\
\text { CHD; it showed } \\
\text { advantage over } \\
\text { traditional } \\
\text { measures }\end{array}$ \\
\hline $\begin{array}{l}\text { Märdby } \\
\text { et al., } \\
2016^{39}\end{array}$ & $\begin{array}{l}\text { 1. Identify adherence } \\
\text { trajectories of } \\
\text { antidepressants } \\
\text { 2. Identify associated } \\
\text { factors }\end{array}$ & \begin{tabular}{|l|} 
Patients aged \\
$18-85$ years
\end{tabular} & Citalopram & $\begin{array}{c}24 \\
\text { months }\end{array}$ & Sweden & 54,248 & MPR & $\begin{array}{l}\text { 1. Perfect } \\
\text { adherence } \\
\text { 2. Moderate } \\
\text { decline } \\
\text { 3. Rapid } \\
\text { decline, } \\
\text { then } \\
\text { increase } \\
\text { 4. Rapid } \\
\text { decline } \\
\text { 5. Very rapid } \\
\text { decline }\end{array}$ & - & $\begin{array}{l}\text { 1. GBTM } \\
\text { identified } \\
5 \text { distinct } \\
\text { patterns } \\
\text { 2. Low } \\
\text { adherence } \\
\text { trajectories } \\
\text { associated } \\
\text { with lower } \\
\text { SEP }\end{array}$ \\
\hline
\end{tabular}




\section{TABLE 1 Summary of Identified Studies (continued)}

\begin{tabular}{|c|c|c|c|c|c|c|c|c|c|c|}
\hline Authors & Objectives & Population & $\begin{array}{c}\text { Type of } \\
\text { Treatment }\end{array}$ & $\begin{array}{c}\text { Length } \\
\text { of Study } \\
\text { Period } \\
\end{array}$ & Country & $\begin{array}{c}\text { Sample } \\
\text { Size }\end{array}$ & $\begin{array}{c}\text { Adherence } \\
\text { Measure }\end{array}$ & $\begin{array}{l}\text { Trajectory } \\
\text { Group }\end{array}$ & $\begin{array}{l}\text { Clinical } \\
\text { Outcomes }\end{array}$ & Conclusions \\
\hline $\begin{array}{l}\text { Lo-Ciganic } \\
\text { et al., } \\
2016^{25}\end{array}$ & $\begin{array}{l}\text { 1. Identify adherence } \\
\text { trajectories of oral } \\
\text { hypoglycemic and } \\
\text { associated factors } \\
\text { 2. Examine association } \\
\text { with clinical events }\end{array}$ & $\begin{array}{l}\text { Patients aged } \\
18-64 \text { years } \\
\text { with DM }\end{array}$ & $\begin{array}{l}\text { Oral hypo- } \\
\text { glycemic } \\
\text { medication }\end{array}$ & 1 year & $\begin{array}{l}\text { United } \\
\text { States }\end{array}$ & 16,256 & $\begin{array}{l}\text { PDC: } \text { used } \\
\text { to compare } \\
\text { GBTM in } \\
\text { the ability } \\
\text { to predict } \\
\text { outcome }\end{array}$ & 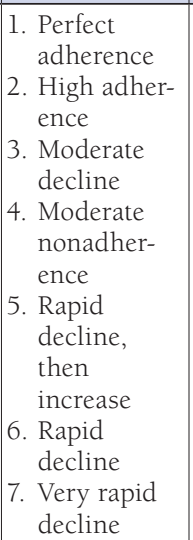 & $\begin{array}{l}\text { 1. DM related } \\
\text { ED visit/ } \\
\text { hospitaliza- } \\
\text { tion } \\
\text { 2. All cause ED } \\
\text { visit/hospi- } \\
\text { talization }\end{array}$ & $\begin{array}{l}\text { Lower } \\
\text { adherence } \\
\text { trajectories } \\
\text { associated with } \\
\text { higher ED/ } \\
\text { hospitalization } \\
\text { events }\end{array}$ \\
\hline $\begin{array}{l}\text { Winn } \\
\text { et al., } \\
2016^{26}\end{array}$ & $\begin{array}{l}\text { 1. Identify adherence } \\
\text { trajectories of ET } \\
\text { 2. Identify associated } \\
\text { factors } \\
\text { 3. Examine association } \\
\text { with mortality }\end{array}$ & $\begin{array}{l}\text { Women with } \\
\text { breast cancer }\end{array}$ & ET & 1 year & $\begin{array}{l}\text { United } \\
\text { States }\end{array}$ & 9,492 & $\begin{array}{l}\text { PDC: } \text { used } \\
\text { to compare } \\
\text { GBTM in } \\
\text { the ability } \\
\text { to predict } \\
\text { outcome }\end{array}$ & \begin{tabular}{|l|} 
1. Perfect \\
adherence \\
2. Slow decline \\
3. Rapid \\
decline, \\
then \\
increase \\
4. Moderate \\
decline \\
5. Rapid \\
decline
\end{tabular} & $\begin{array}{l}\text { All-cause } \\
\text { mortality }\end{array}$ & $\begin{array}{l}\text { Low adherence } \\
\text { groups } \\
\text { associated with } \\
\text { higher mortality } \\
\text { rate }\end{array}$ \\
\hline $\begin{array}{l}\text { Chen } \\
\text { et al., } \\
2016^{42}\end{array}$ & $\begin{array}{l}\text { 1. Identify adherence } \\
\text { trajectories of hypo- } \\
\text { glycemic } \\
\text { 2. Identify associated } \\
\text { factors }\end{array}$ & $\begin{array}{l}\text { Patients aged } \\
\geq 18 \text { years } \\
\text { type } 2 \\
\text { diabetes }\end{array}$ & $\begin{array}{l}\text { Oral hypo- } \\
\text { glycemic } \\
\text { medication }\end{array}$ & 6 years & Taiwan & 12,123 & MPR & \begin{tabular}{|l} 
1. \\
Perfect \\
adherence \\
2. Moderate \\
adher- \\
ence, then \\
increase \\
3. Moderate \\
decline \\
4. Low adher- \\
ence, then \\
increase
\end{tabular} & - & $\begin{array}{l}\text { GBTM help } \\
\text { in identifying } \\
\text { heterogeneity } \\
\text { of medication } \\
\text { adherence }\end{array}$ \\
\hline $\begin{array}{l}\text { Franklin } \\
\text { et al., } \\
2015^{27}\end{array}$ & $\begin{array}{l}\text { Identify adherence } \\
\text { trajectories of statin }\end{array}$ & $\begin{array}{l}\text { Patients aged } \\
\geq 65 \text { years }\end{array}$ & Statins & 1 year & $\begin{array}{l}\text { United } \\
\text { States }\end{array}$ & 77,703 & PDC & $\begin{array}{l}\text { 1. } \text { Perfect } \\
\text { adherence } \\
\text { 2. Moderate } \\
\text { adherence } \\
\text { 3. Rapid } \\
\text { decline, } \\
\text { then } \\
\text { increase } \\
\text { 4. Moderate } \\
\text { decline, } \\
\text { then } \\
\text { increase } \\
\text { 5. Rapid } \\
\text { decline } \\
\text { 6. Very rapid } \\
\text { decline }\end{array}$ & - & $\begin{array}{l}\text { Initial } \\
\text { adherence } \\
\text { behavior } \\
\text { associated with } \\
\text { better future } \\
\text { adherence }\end{array}$ \\
\hline
\end{tabular}




\section{TABLE 1 Summary of Identified Studies (continued)}

\begin{tabular}{|c|c|c|c|c|c|c|c|c|c|c|}
\hline Authors & Objectives & Population & $\begin{array}{c}\text { Type of } \\
\text { Treatment }\end{array}$ & $\begin{array}{l}\text { Length } \\
\text { of Study } \\
\text { Period }\end{array}$ & Country & $\begin{array}{c}\text { Sample } \\
\text { Size }\end{array}$ & $\begin{array}{c}\text { Adherence } \\
\text { Measure }\end{array}$ & $\begin{array}{l}\text { Trajectory } \\
\text { Group }\end{array}$ & $\begin{array}{l}\text { Clinical } \\
\text { Outcomes }\end{array}$ & Conclusions \\
\hline $\begin{array}{l}\text { Newman- } \\
\text { Casey } \\
\text { et al., } \\
2015^{28}\end{array}$ & $\begin{array}{l}\text { 1. Identify adherence } \\
\text { trajectories of glau- } \\
\text { coma medication } \\
\text { 2. Identify associated } \\
\text { factors }\end{array}$ & \begin{tabular}{|l|} 
Patients aged \\
$\geq 40$ years \\
treated for \\
glaucoma
\end{tabular} & $\begin{array}{l}\text { Glaucoma } \\
\text { medication }\end{array}$ & 4 years & $\begin{array}{l}\text { United } \\
\text { States }\end{array}$ & 1,234 & MPR & 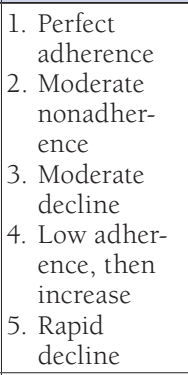 & - & $\begin{array}{l}\text { Adherence } \\
\text { patterns for } \\
\text { first year, had } \\
\text { great impact on } \\
\text { future adher- } \\
\text { ence behavior }\end{array}$ \\
\hline $\begin{array}{l}\text { Juarez } \\
\text { et al., } \\
2015^{29}\end{array}$ & $\begin{array}{l}\text { 1. Identify adherence } \\
\text { trajectories of ACEI } \\
\text { 2. Identify associated } \\
\text { factors }\end{array}$ & $\begin{array}{l}\text { Patients with } \\
\text { CHF }\end{array}$ & ACEI & 6 years & $\begin{array}{l}\text { United } \\
\text { States }\end{array}$ & 10,986 & MPR & $\begin{array}{l}\text { 1. } \text { Perfect } \\
\text { adherence } \\
\text { 2. Moderate } \\
\text { decline } \\
\text { 3. Low adher- } \\
\text { ence, then } \\
\text { increase } \\
\text { 4. Rapid } \\
\text { decline }\end{array}$ & - & $\begin{array}{l}\text { Patient factors } \\
\text { associated with } \\
\text { low adherence } \\
\text { trajectories } \\
\text { can be used } \\
\text { to target } \\
\text { interventions }\end{array}$ \\
\hline $\begin{array}{l}\text { Franklin } \\
\text { et al., } \\
2015^{30}\end{array}$ & $\begin{array}{l}\text { 1. Identify adherence } \\
\text { trajectories of statin } \\
\text { 2. Examine association } \\
\text { with clinical events }\end{array}$ & \begin{tabular}{|l|} 
Patients \\
in the \\
UnitedHealth \\
Optum \\
Research \\
Datamart, \\
aged 35-64 \\
years
\end{tabular} & Statins & 1 year & $\begin{array}{l}\text { United } \\
\text { States }\end{array}$ & 519,842 & $\begin{array}{l}\text { PDC: used } \\
\text { cross- } \\
\text { validation } \\
\text { methods to } \\
\text { ascertain } \\
\text { if initial } \\
\text { PDC could } \\
\text { predict } \\
\text { trajectory } \\
\text { member- } \\
\text { ship }\end{array}$ & $\begin{array}{l}\text { 1. Perfect } \\
\text { adherence } \\
\text { 2. Rapid } \\
\text { decline then } \\
\text { increase } \\
\text { 3. Moderate } \\
\text { decline } \\
\text { 4. Moderate } \\
\text { decline, } \\
\text { then } \\
\text { increase } \\
\text { 5. Rapid } \\
\text { decline } \\
\text { 6. Very rapid } \\
\text { decline }\end{array}$ & $\begin{array}{l}\text { Hospitalization } \\
\text { for an acute } \\
\text { coronary event, } \\
\text { revasculariza- } \\
\text { tion, cerebro- } \\
\text { vascular event, } \\
\text { or heart failure }\end{array}$ & $\begin{array}{l}\text { Adherence } \\
\text { trajectories } \\
\text { predicts future } \\
\text { clinical out- } \\
\text { comes better } \\
\text { than PDC }\end{array}$ \\
\hline $\begin{array}{l}\text { Franklin } \\
\text { et al., } \\
201310\end{array}$ & $\begin{array}{l}\text { 1. Identify adherence } \\
\text { trajectories of statin } \\
\text { 2. Identify associated } \\
\text { factors }\end{array}$ & \begin{tabular}{|l|} 
Patients \\
initiating a \\
statin in CVS \\
Caremark
\end{tabular} & Statins & $\begin{array}{c}15 \\
\text { months }\end{array}$ & $\begin{array}{l}\text { United } \\
\text { States }\end{array}$ & 264,789 & PDC & 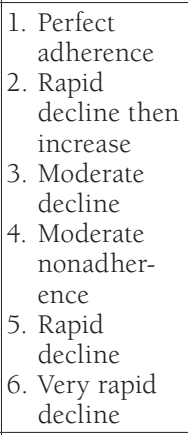 & - & $\begin{array}{l}\text { GBTM summa- } \\
\text { rized adherence } \\
\text { patterns better } \\
\text { than traditional } \\
\text { measures }\end{array}$ \\
\hline $\begin{array}{l}\text { Vadhariya } \\
\text { et al., } \\
2019^{33}\end{array}$ & $\begin{array}{l}\text { 1. Identify distinct } \\
\text { trajectories of } \\
\text { adherence to statin } \\
\text { therapy } \\
\text { 2. Identify } \\
\text { sociodemographic } \\
\text { and clinical predictors } \\
\text { of trajectory } \\
\text { membership }\end{array}$ & $\begin{array}{l}\text { Patients } \\
\text { enrolled in } \\
\text { a Medicare } \\
\text { Advantage } \\
\text { Plan }\end{array}$ & Statins & $\begin{array}{c}12 \\
\text { months }\end{array}$ & $\begin{array}{l}\text { United } \\
\text { States }\end{array}$ & 7,850 & PDC & $\begin{array}{l}\text { 1. High/nearly } \\
\text { perfect } \\
\text { adherence } \\
\text { 2. Declining } \\
\text { adherence, } \\
\text { then } \\
\text { increase } \\
\text { 3. Gradual } \\
\text { decline } \\
\text { 4. Rapid } \\
\text { decline }\end{array}$ & - & $\begin{array}{l}\text { Adherence } \\
\text { trajectories are } \\
\text { consistent with } \\
\text { previous find- } \\
\text { ings in the lit- } \\
\text { erature; patient } \\
\text { characteristics } \\
\text { are found to be } \\
\text { predictors of } \\
\text { trajectory } \\
\text { membership }\end{array}$ \\
\hline
\end{tabular}




\section{TABLE 1 Summary of Identified Studies (continued)}

\begin{tabular}{|c|c|c|c|c|c|c|c|c|c|c|}
\hline Authors & Objectives & Population & $\begin{array}{c}\text { Type of } \\
\text { Treatment }\end{array}$ & $\begin{array}{c}\text { Length } \\
\text { of Study } \\
\text { Period }\end{array}$ & Country & $\begin{array}{c}\text { Sample } \\
\text { Size }\end{array}$ & $\begin{array}{c}\text { Adherence } \\
\text { Measure }\end{array}$ & $\begin{array}{c}\text { Trajectory } \\
\text { Group }\end{array}$ & $\begin{array}{l}\text { Clinical } \\
\text { Outcomes }\end{array}$ & Conclusions \\
\hline $\begin{array}{l}\text { Lambert- } \\
\text { Cote, } \\
\text { et al. } \\
2020^{40}\end{array}$ & $\begin{array}{l}\text { 1. Identify adherence } \\
\text { trajectories and } \\
\text { characterize trajectory } \\
\text { shapes } \\
\text { 2. Identify factors } \\
\text { associated with group } \\
\text { membership }\end{array}$ & $\begin{array}{l}\text { Women with } \\
\text { breast can- } \\
\text { cer living in } \\
\text { metropolitan } \\
\text { areas covered } \\
\text { by National } \\
\text { Health } \\
\text { Insurance } \\
\text { plan }\end{array}$ & $\begin{array}{l}\text { Adjuvant } \\
\text { endocrine } \\
\text { therapy }\end{array}$ & 5 years & France & 674 & PDC & \begin{tabular}{|l} 
1. Very high \\
adherence \\
2. High adher- \\
ence \\
3. Slow decline \\
4. Moderate \\
decline \\
5. Quick \\
decline
\end{tabular} & - & $\begin{array}{l}\text { Previous expo- } \\
\text { sure to chemo- } \\
\text { therapy and } \\
\text { personalized } \\
\text { care plan were } \\
\text { found to be pre- } \\
\text { dictors of trajec- } \\
\text { tory member- } \\
\text { ship; dynamic } \\
\text { behavior of } \\
\text { adherence not } \\
\text { fully captured } \\
\text { by traditional } \\
\text { annualization of } \\
\text { PDC }\end{array}$ \\
\hline $\begin{array}{l}\text { Ajrouche } \\
\text { et al., } \\
2020^{41}\end{array}$ & $\begin{array}{l}\text { 1. Identify adherence } \\
\text { trajectories of low- } \\
\text { dose aspirin } \\
\text { 2. Describe trajectories } \\
\text { based on primary or } \\
\text { secondary indication } \\
\text { for low-dose aspirin } \\
\text { 3. Identify predictors } \\
\text { of low-dose aspirin } \\
\text { adherence trajectory } \\
\text { membership }\end{array}$ & $\begin{array}{l}\text { Patients with } \\
\text { at least } 3 \\
\text { months of } \\
\text { follow-up } \\
\text { after first } \\
\text { low-dose } \\
\text { aspirin } \\
\text { delivery }\end{array}$ & $\begin{array}{l}\text { Low-dose } \\
\text { aspirin }\end{array}$ & 3 years & France & 11,793 & PDC & $\begin{array}{l}\text { 1. Perfect } \\
\text { adherence } \\
\text { 2. Declining } \\
\text { adherence } \\
\text { 3. Declining } \\
\text { adherence } \\
\text { then } \\
\text { increase } \\
\text { 4. Low } \\
\text { adherence }\end{array}$ & $\begin{array}{l}\text { Hemorrhagic } \\
\text { events }\end{array}$ & $\begin{array}{l}\text { GBTM provides } \\
\text { a better under- } \\
\text { standing of lon- } \\
\text { gitudinal trends } \\
\text { by yielding } \\
\text { visual develop- } \\
\text { mental trajecto- } \\
\text { ries; nonadher- } \\
\text { ence trajectories } \\
\text { seem to be } \\
\text { associated with } \\
\text { uncertain effi- } \\
\text { cacy of using } \\
\text { low-dose aspi- } \\
\text { rin in primary } \\
\text { prevention }\end{array}$ \\
\hline $\begin{array}{l}\text { Hickson } \\
\text { et al., } \\
2020^{32}\end{array}$ & $\begin{array}{l}\text { Identify number of } \\
\text { adherence trajectories } \\
\text { in statin users in the } 6 \\
\text { months before an acute } \\
\text { myocardial infarction. }\end{array}$ & $\begin{array}{l}\text { Medicare } \\
\text { beneficiaries }\end{array}$ & Statins & $\begin{array}{c}6 \\
\text { months }\end{array}$ & $\begin{array}{l}\text { United } \\
\text { States }\end{array}$ & 113,296 & PDC & $\begin{array}{l}\text { 1. Persistently } \\
\text { adherent } \\
\text { 2. Moderately } \\
\text { adherent } \\
\text { 3. Declining } \\
\text { adherence } \\
\text { 4. Persistently } \\
\text { nonadherent }\end{array}$ & - & $\begin{array}{l}\text { GBTM com- } \\
\text { prises subjec- } \\
\text { tive decisions } \\
\text { to assess } \\
\text { longitudinal } \\
\text { trends in adher- } \\
\text { ence behavior; } \\
\text { including } \\
\text { spaghetti plots } \\
\text { to confirm the } \\
\text { adequacy of } \\
\text { trajectory group } \\
\text { membership } \\
\text { may improve } \\
\text { internal validity }\end{array}$ \\
\hline $\begin{array}{l}\text { Zongo } \\
\text { et al., } \\
2019^{45}\end{array}$ & $\begin{array}{l}\text { Identify number of } \\
\text { adherence trajectories } \\
\text { before and after acute } \\
\text { coronary syndrome or } \\
\text { stroke. }\end{array}$ & $\begin{array}{l}\text { Patients } \\
\text { hospitalized } \\
\text { for acute } \\
\text { coronary } \\
\text { syndrome }\end{array}$ & $\begin{array}{l}\text { Lipid- } \\
\text { lowering } \\
\text { drugs }\end{array}$ & $\begin{array}{l}2 \text { years } \\
\text { (1 year } \\
\text { before } \\
\text { and } 1 \\
\text { year } \\
\text { after })\end{array}$ & Canada & 10,623 & PDC & 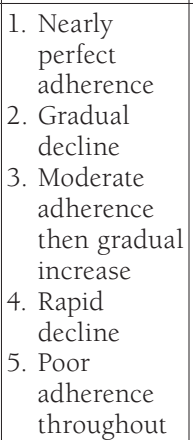 & - & $\begin{array}{l}\text { The occurrence } \\
\text { of acute coro- } \\
\text { nary syndrome } \\
\text { or stroke seems } \\
\text { to influence } \\
\text { continued } \\
\text { behavior of } \\
\text { adherence }\end{array}$ \\
\hline
\end{tabular}


TABLE 1 Summary of Identified Studies (continued)

\begin{tabular}{|c|c|c|c|c|c|c|c|c|c|c|}
\hline Authors & Objectives & Population & $\begin{array}{c}\text { Type of } \\
\text { Treatment }\end{array}$ & $\begin{array}{c}\text { Length } \\
\text { of Study } \\
\text { Period }\end{array}$ & Country & $\begin{array}{c}\text { Sample } \\
\text { Size }\end{array}$ & $\begin{array}{c}\text { Adherence } \\
\text { Measure }\end{array}$ & $\begin{array}{c}\text { Trajectory } \\
\text { Group }\end{array}$ & $\begin{array}{c}\text { Clinical } \\
\text { Outcomes } \\
\end{array}$ & Conclusions \\
\hline $\begin{array}{l}\text { Hernandez } \\
\text { et al., } \\
2019^{34}\end{array}$ & $\begin{array}{l}\text { Identify number of } \\
\text { adherence trajectories of } \\
\text { patients about to initiate } \\
\text { oral anticoagulants for } \\
\text { atrial fibrillation }\end{array}$ & $\begin{array}{l}\text { Patients } \\
\text { diagnosed } \\
\text { with atrial } \\
\text { fibrillation }\end{array}$ & $\begin{array}{l}\text { Oral } \\
\text { anti- } \\
\text { coagulants }\end{array}$ & $\begin{array}{c}12 \\
\text { months }\end{array}$ & $\begin{array}{l}\text { United } \\
\text { States }\end{array}$ & 34,898 & PDC & $\begin{array}{l}\text { 1. Perfect } \\
\text { adherence } \\
\text { 2. Gradual } \\
\text { decline } \\
\text { 3. Initial low } \\
\text { adher- } \\
\text { ence then } \\
\text { increase } \\
\text { 4. Consistently } \\
\text { poor } \\
\text { adherence }\end{array}$ & - & $\begin{array}{l}\text { Patients at } \\
\text { higher risk of } \\
\text { bleeding or } \\
\text { stroke are more } \\
\text { likely to go } \\
\text { through poorer } \\
\text { adherence tra- } \\
\text { jectories; late } \\
\text { initiators are } \\
\text { also more likely } \\
\text { to belong to } \\
\text { nonadherence } \\
\text { trajectories }\end{array}$ \\
\hline $\begin{array}{l}\text { Tervonen } \\
\text { et al., } \\
2019^{44}\end{array}$ & $\begin{array}{l}\text { Identify adherence } \\
\text { trajectories and } \\
\text { treatment and patient } \\
\text { characteristics } \\
\text { associated with } \\
\text { discontinuation and } \\
\text { restart of therapy. }\end{array}$ & $\begin{array}{l}\text { Women } \\
\text { diagnosed } \\
\text { with HER-2 } \\
\text { positive } \\
\text { breast cancer }\end{array}$ & ET & 5 years & Australia & 2,656 & MPR & $\begin{array}{l}\text { 1. Perfect } \\
\text { adherence } \\
\text { 2. Stable with } \\
\text { late decline } \\
\text { 3. Moderate } \\
\text { decline } \\
\text { 4. Quick } \\
\text { decline then } \\
\text { stable } \\
\text { 5. Quick } \\
\text { decline }\end{array}$ & $\begin{array}{l}\text { Discontinuation } \\
\text { and restart of } \\
\text { therapy }\end{array}$ & $\begin{array}{l}\text { Consistent tra- } \\
\text { jectories were } \\
\text { found; GBTM } \\
\text { analysis allows } \\
\text { the identifica- } \\
\text { tion of critical } \\
\text { time points that } \\
\text { may warrant } \\
\text { intervention: } \\
6 \text { months, } 1.5 \\
\text { years, and } 4 \\
\text { years after } \\
\text { initiation }\end{array}$ \\
\hline
\end{tabular}

$A C E=$ angiotensin-converting enzyme; $A C E I=$ angiotensin-converting enzyme inhibitor; $A R B=$ angiotensin receptor blockers; $B P=b l o o d$ pressure; $C H D=$ coronary heart disease; $\mathrm{CHF}=$ congestive heart failure; $D M=$ diabetes mellitus; $E D=$ emergency department; $E H R=$ electronic health record; $E T=$ endrocrine therapy; $F D C=$ fixed dose combination; GBTM = group-based trajectory modeling; GP=general practitioner; HCQ =hydroxychloroquine; $M P R=$ medication possession ratio; $P D C=$ proportion of days covered; SEP=socioeconomic position; SSRI= selective serotonin reuptake inhibitor.

adherence was poor at the beginning of the study period but would gradually improve over time $(\mathrm{n}=18,64.3 \%){ }^{10,19,20,23-30 \text {, }}$ 33,37-39,41,42,45 This rebound in adherence was more frequent following a period of rapid declining adherence ( $n=12,42.9 \%$ ), although 5 studies identified trajectories with improving medication adherence following rapid and gradual declines. ${ }^{19,23,27,30,34}$ This trajectory was different from the others that appeared to display an initial pattern of nonadherence, which evolved to a pattern of increasing adherence. Despite not being referenced in the included studies, since most used data from administrative claims, this transition may be indicative of some event or intervention that explains the change in the adherence patterns of patients.

In studies with a follow-up period between 9 and 18 months, there was a clear pattern in the time point at which an increase would be observable in the declining adherence trajectory (4-6 months). $10,20,23-27,30,33,34,37,38,45$ The studies with longer follow-up periods (3-6 years) described increases at different time points, primary between the first and second year. ${ }^{26,40,41,44}$ The type of drug being analyzed also varied, including statins, antipsychotics, antidepressants, and angiotensin-converting enzyme inhibitors, which does not indicate a common underlying reason for the increase in adherence after a period of declining adherence. ${ }^{28,41,42,44}$ In a study with a follow-up period of 5 years, a declining adherence trajectory increased to a stable level of adherence, suggesting that a trend of reaching a plateau in adherence may be observed if follow-up periods were extended beyond 18 months. ${ }^{44}$ It is possible that the trend of reaching a plateau in adherence would be observed in the shorter follow-up period studies.

\section{Drug Classes Studied}

With the exception of 1 study, ${ }^{38}$ all studies focused on adherence to a single drug class over time. Single drug class studies examined trajectories of a wide selection of medications: antihypertensives, statins, oral antihyperglycemic drugs, antipsychotics, antidepressants, treatment for systematic lupus erythematosus, glaucoma, and endocrine therapy (Table 1).

Four of the 6 studies that focused exclusively on statin adherence identified 6 adherence trajectories, while the other 2 found only 4 trajectories. Studies that examined statins and antihypertensives, however, varied in the number of identified trajectories (3-6 trajectories), as did studies that included oral antidiabetic agents (4-7 trajectories). 


\section{Patient Characteristics Associated with Trajectories}

Patient sociodemographic and clinical characteristics were commonly associated with the likelihood of belonging to specific adherence trajectories in the included studies. Juarez et al. (2015) found that race, (e.g., black, Asian, and Pacific Islanders) was associated with belonging to nonadherence trajectories when compared with white patients. ${ }^{29}$ The same types of associations were found in other studies in which individuals who were younger, nonwhite, males, with more severe disease and comorbidities, and with lower educational attainment and socioeconomic status were more likely to follow low adherence trajectories. ${ }^{10,25,26,28,29,33,39,42,46}$

Conflicting results were found in 2 studies, in which females were more likely to belong to nonadherence trajectories. ${ }^{33,41}$ Low adherence trajectories were found for patients from a lower socioeconomic status s, 30,32 and for individuals with more severe diseases and comorbidities. ${ }^{24,46}$ Treatment complexity, race, previous history of the disease, frailty, concomitant use of opioids, and comorbidities were also associated with lower or nonadherence trajectories. ${ }^{22-26,28,35,37-39,46}$ Adherence trajectories were influenced by the experiences that patients had with taking other medications. MacEwan et al. (2018) found that adherence to atypical antipsychotic drugs can be a predictor of adherence to other medications. ${ }^{20}$ Previous medication use was also found to be a predictor of higher adherence in other studies. ${ }^{23,35,43}$

Patients in groups defined by declining adherence followed by an increase seem to have particular features. Although most studies found typical sociodemographic characteristics in patients in nonadherence trajectories, drug abuse, alcoholism, ${ }^{24}$ hypertension, ${ }^{23}$ a higher number of comorbid diagnoses, ${ }^{38}$ and higher levels of out-of-pocket expenditures ${ }^{38}$ were each reported to be overrepresented in declining adherence or nonadherence trajectories. In contrast, other characteristics seemed to have made patients less likely to belong to declining adherence trajectories or declining adherence followed by increasing trajectories. These characteristics included higher adherence to previous medication and being female outside of the labor market. ${ }^{20,37}$

Finally, the only study with a pre/post approach found that patients who were previously in a declining followed by increase in adherence trajectory were more likely to belong to nearly perfect adherence trajectories after a critical medical event (i.e., acute coronary syndrome). ${ }^{41}$

\section{Trajectories and Health Care Resource Utilization and Health Outcomes}

Five studies examined and found a relationship between adherence trajectories and health care events (e.g., hospitalization events, emergency department [ED] visits, adverse events, or death), 24-26,30,36 reporting that consistent, high adherence trajectories were associated with lower health care resource utilization and improved health outcomes. Specifically, fewer hospitalizations were seen among patients in consistent, high adherence trajectories for antihypertensives, ${ }^{36}$ statins, ${ }^{30}$ and oral antidiabetic drugs, ${ }^{25}$ with similar trends for reductions in ED visits in studies on antipsychotics and oral antidiabetics. ${ }^{24,25}$ More use of health care resources was not always associated with belonging to poor adherence trajectories. Although not specific to increased health care resource utilization, a more individualized approach to treatment plans seemed to improve the outlook of medication adherence. In Lambert-Côté et al. (2020), women with breast cancer who had received a personalized treatment plan that comprised receiving a written document with information and resources available throughout the treatment duration provided at diagnosis were more likely to belong to consistent, high adherence trajectories. ${ }^{40}$

Conversely, patients in consistently nonadherent or declining adherence trajectories had a significantly increased risk of adverse outcomes. Patients on endocrine therapy in the rapidly and slowly declining adherence trajectories had a significantly higher risk of death compared with those in perfect adherence trajectories. ${ }^{19}$ Consistent nonadherence trajectory group membership was associated with a higher risk of cardiovascular events (e.g., hospitalization for acute coronary event, revascularization, cerebrovascular event, or heart failure) in patients taking statins. ${ }^{30}$

\section{Predictive Validity of GBTM}

Five studies compared GBTM with conventional dichotomous measures (e.g., PDC), and all concluded that GBTM is advantageous, namely, because of the ability to encapsulate the trends of medication adherence over a period of time. . $3,25,26,30,37^{\text {Of }}$ these, 3 studies used multinomial logistic regression and computed the c-statistic to compare predictive validity of using GBTM versus dichotomized periodic PDC. ${ }^{23,25,26}$ However, the comparison in each study focused on different aspects. Hargrove et al. (2017) reported that a 6-group trajectory model better distinguished between adherent and nonadherent months than either PDC or even proportion of months covered (another possession-based adherence measure). ${ }^{23}$ Lo-Ciganic et al. (2016) reported the c-statistic to compare dichotomized PDC and group-based trajectory models in predicting diabetesrelated hospitalization and ED visits. ${ }^{25}$ In Franklin et al. (2015), the c-statistic was used to compare the group-based trajectory model and dichotomized PDC in predicting cardiovascular events. In all situations, the c-statistic of GBTM fared better than the traditional dichotomized PDC $\geq 80 \%$ threshold approach. ${ }^{30}$ While the other 2 studies did not use multinomial logistic regression to compare GBTM to the traditional PDC $>80 \%$ approach, the respective authors argued that GBTM provided a more refined approach in identifying longitudinal trends. Aarnio et al. (2016) found GBTM to be comparable with PDC in identifying relationships between nonadherence and 


\section{FIGURE 2 Illustration of Similar Medication Adherence Trajectories}

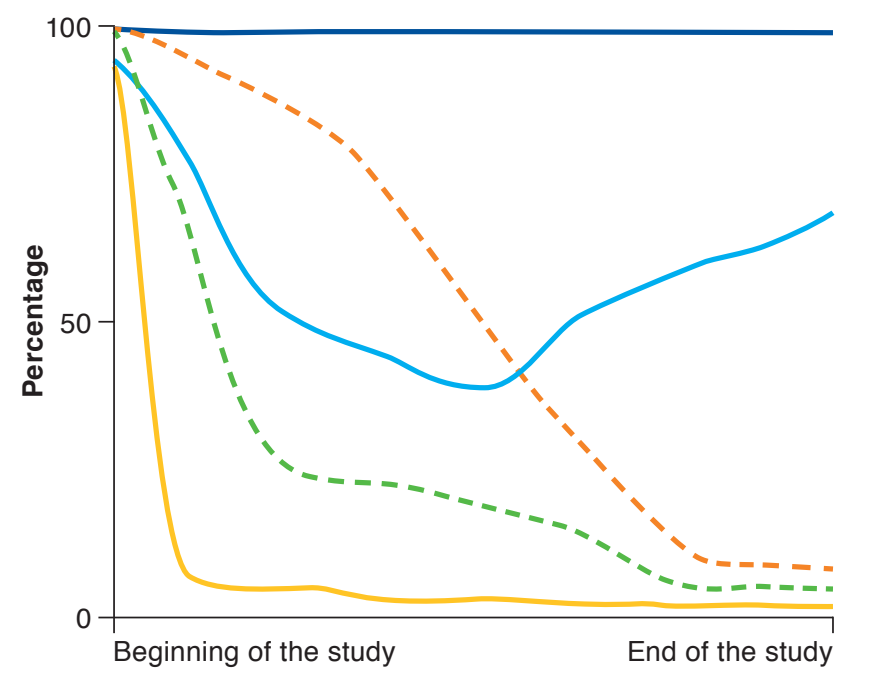

\begin{tabular}{cc}
\hline Consistent, high adherence & --- Declining adherence (slow) \\
--- Decling adherence (fast) & Early and consistent \\
Initial nonadherence & nonadherence \\
followed by an increase &
\end{tabular}

lower socioeconomic status, but that GBTM provided a more differentiating approach to analyzing nonadherent groups. ${ }^{37}$ Winn et al. (2016) also determined that GBTM better differentiated adherence groups. ${ }^{26}$

\section{Discussion}

GBTM has gained traction in adherence research based on its ability to better capture the temporal and fluid patterns of medication adherence compared with static, conventional measures. This review found that 4 to 6 trajectory groups could be used in most cases to describe medication-taking behavior over time. The most commonly identified trajectories can be described using the following labels: (a) consistent, high adherence, (b) declining adherence, (c) consistent nonadherence, and (d) initial nonadherence followed by an increase. Studies that identified fewer or more trajectories than 4 to 6 may have been affected by high adherence of patients studied, bias in selecting study subjects, the nature of the drugs studied, unique characteristics of patient populations, and differences in the rate of decline from adherence to nonadherence..$^{25,36}$ Figure 2 shows the general trend identified in this literature review. Generally, adherence trajectories can be summarized by 4 trajectory groups, with the declining adherence trajectory possibly occurring at different rates (fast and slow).
The value provided by GBTM over current measures such as PDC and MPR is that it describes behavior over time instead of at discrete time periods. Understanding the temporal changes in behavior is important in evaluating and predicting influences on patient adherence. Hypothetically, a patient who interrupts medication taking because of complications might have a different trajectory than the patient who stops because of an issue of affordability or other complication, assuming that affordability and complications happen at different time points. Rather than classifying patients into dichotomous adherent and nonadherent groups, GBTM provides the longitudinal perspective to understand and describe patterns of nonadherence.

The distillation of adherence trajectories has the power to differentiate between underlying causes of nonadherence in a way that periodic dichotomization of PDC cannot. Early nonadherence can be a result of adverse events that otherwise would not be identified if the patient did not follow-up with a health care professional. ${ }^{20,23,43,46}$ Similarly, declining adherence followed by an increase, may be related to specific medical events that resulted in increased motivation to resume the treatment plan, suggesting that targeted interventions following medical events may be effective in improving adherence in these patients. ${ }^{41}$ These patterns should be explored through further research into the causes and potential solutions to nonadherence trajectory groups.

When contrasted with dichotomous PDC and MPR measures, GBTM offers a richer description of patient behavior. In Märdby et al. (2016), the simple dichotomized PDC analysis showed that $50 \%$ of the patient population was nonadherent. ${ }^{39}$ However, when a GBTM analysis was conducted, they found that a significant portion of the patients initially classified as nonadherent actually belonged to group with declining adherence that later increased. The finding of a subgroup with GBTM suggests heterogeneity among nonadherents that requires a more sophisticated array of interventions to improve medication adherence.

Other implications result from this review. Payers such as the Centers for Medicare \& Medicaid Services and other private health insurers provide discounts and rebates for providers who meet certain quality measures, such as medication adherence. Specifically, Medicare Part D plans incorporate medication adherence as a significant component of their quality-based payment program (Medicare star ratings). ${ }^{47,48}$ In 1 study, 2 trajectories (declining followed by an increase and slow decline) were found to have no difference in risk of mortality when compared with the consistent, high adherence trajectory. ${ }^{26}$ Therefore, it is likely that further research may elicit other optimal thresholds of adherence. Thus, systems that either reward or penalize providers based on medication adherence measures should use methods that appropriately demonstrate variations in medication adherence. 
Moreover, GBTM has already served to develop a tailored motivational interviewing system based on adherence trajectories. The individualization of interventions not only allows for a better use of available resources but is able to produce significant improvements in adherence. ${ }^{49}$ Likewise, using GBTM may also allow the identification of patients who are keen on following a specific adherence trajectory and, consequently, develop an action plan to invert the trend. Future research should clarify significant thresholds of medication adherence improvements that result in improvement of patient outcomes, as opposed to computing an average medication adherence percentage in a given period.

\section{Limitations}

Some limitations were identified in the studies in this review. It was apparent that some of the studies used different criteria to fit the final models. For example, despite the criteria for model fit, 1 study forced models ranging from 1 to 4 trajectory groups. ${ }^{42}$ This lack of consistency of the criteria used in the GBTM method can skew the overall conclusion of how many adherence groups exist in the general population.

Another limitation lies in the measures used to calculate GBTM, which is typically PDC. Adherence using PDC is assessed through pharmacy-paid claim data. Prescription fill records only indicate that a patient received the medication but does not indicate if the medication was taken as directed. In addition, PDC does not measure primary medication adherence, which happens when newly prescribed medications are never obtained by the patient. Therefore, analyzing adherence using pharmacy-paid claims data does not display patients who never really fill their prescriptions.

Finally, study heterogeneity could introduce bias in this review. Studies varied by their length of time (12 months to 24 months), number and types of medicines examined, practice setting, patient population, and more. Any of these variables can bias study outcomes in a way that masks meaningful results.

\section{Conclusions}

The findings of this review provide a summary of the evidence about patterns of medication adherence, and they show consistency with what is currently known. In most studies, a consistently adherent trajectory group was found, which typically made up a significant portion of the study sample. While previous literature reports that $50 \%$ of patients are typically adherent with their medications, ${ }^{1,13}$ it is clear that using a GBTM approach provides a richer perspective of patient adherence behavior. Adherence in patient populations in this study also found demographic and other variables associated with adherence found in the literature. Low adherence trajectories were commonly associated with low socioeconomic status, being nonwhite, and low health care utilization.
As more is learned about the number, shape, and causes of trajectories, future research will need to be conducted to identify how health care interventions can influence nonadherence trajectories. Interventions may need to be timed differently for different stages of adherence trajectories. The acuity of care given might also need to vary across different trajectories depending on patient needs or circumstances.

Public policy might also be informed by a better understanding of patient behavior over time. Public and private payers of prescription medications may need to adjust incentives linked to adherence measures. For instance, the CMS Star Ratings Program associated with Medicare Part D insurance plans use medication adherence as a significant component of the quality-based payment program. ${ }^{47,48}$ As shown in this review, it is possible that clinical outcomes (e.g., mortality) are not significantly different between perfect adherence trajectories and other trajectories currently classified as nonadherent (PDC $<80 \%$ ). We conclude that adherence measurement by dichotomization of period PDC alone might lead to unintended consequences or missed opportunities. Future research into this topic is needed to better understand how GBTM can be used to improve medication adherence interventions.

\section{Authors}

MAI ALHAZAMI, BPharm, MSc, PhD, Kuwait University, Jabriya. VASCO M. PONTINHA, MPharm, MA; JULIE A. PATTERSON, PharmD, PhD; and DAVID A. HOLDFORD, PhD, Virginia Commonwealth University School of Pharmacy, Richmond.

AUTHOR CORRESPONDENCE: David A. Holdford, PhD, Professor, Department of Pharmacotherapy and Outcomes Science, VCU School of Pharmacy, Richmond, VA 23298. Tel.: 804.828.6103; Email: daholdfo@vcu.edu.

\section{DISCLOSURES}

No outside funding supported this study. The authors have no conflicts of interest to declare.

\section{REFERENCES}

1. World Health Organization. Adherence to long-term therapies: evidence for action. 2003. Available at: https://www.who.int/chp/knowledge/ publications/adherence_introduction.pdf?ua=1. Accessed July 8, 2020.

2. Khunti K, Seidu S, Kunutsor S, Davies M. Association between adherence to pharmacotherapy and outcomes in type 2 diabetes: a meta-analysis. Diabetes Care. 2017;40(11):1588-96.

3. Cutler RL, Fernandez-Llimos F, Frommer M, Benrimoj C, GarciaCardenas V. Economic impact of medication non-adherence by disease groups: a systematic review. BMJ Open. 2018;8(1):e016982

4. Forbes CA, Deshpande S, Sorio-Vilela F, et al. A systematic literature review comparing methods for the measurement of patient persistence and adherence. Curr Med Res Opin. 2018;34(9):1613-25. 
5. Clifford S, Perez-Nieves M, Skalicky AM, Reaney M, Coyne KS. A systematic literature review of methodologies used to assess medication adherence in patients with diabetes. Curr Med Res Opin. 2014;30(6):1071-85.

6. Stolpe S, Kroes MA, Webb N, Wisniewski T. A systematic review of insulin adherence measures in patients with diabetes. J Manag Care Spec Pharm. 2016;22(11):1224-46. Available at: https://www.jmcp.org/ doi/10.18553/jmcp.2016.22.11.1224.

7. Cranston L, Dixon L. Emerging trends in adherence and quality measurement: what the future holds. Pharmacy Times. August 20, 2014. Available at: https://www.pharmacytimes.com/publications/ directions-in-pharmacy/2014/august2014/emerging-trends-in-adherenceand-quality-measurement-what-the-future-holds. Accessed July 8, 2020.

8. National Quality Forum. NQF-endorsed measures for patient safety technical report. January 30, 2015. Available at: http://www.qualityforum. org/WorkArea/linkit.aspx?LinkIdentifier=id\&ItemID=78776. Accessed July 8, 2020.

9. Karve S, Cleves MA, Helm M, Hudson TJ, West DS, Martin BC. Prospective validation of eight different adherence measures for use with administrative claims data among patients with schizophrenia. Value Health. 2009;12(6):989-95.

10. Franklin JM, Shrank WH, Pakes J, et al. Group-based trajectory models: a new approach to classifying and predicting long-term medication adherence. Med Care. 2013;51(9):789-96.

11. Lee JK, Grace KA, Foster TG, et al. How should we measure medication adherence in clinical trials and practice? Ther Clin Risk Manag. 2007;3(4):685-90.

12. Vrijens B, De Geest S, Hughes DA, et al. A new taxonomy for describing and defining adherence to medications. Br J Clin Pharmacol. 2012;73(5):691-705.

13. Brown MT, Bussell JK. Medication adherence: WHO cares? Mayo Clin Proc. 2011;86(4):304-14.

14. Kronish IM, Ye S. Adherence to cardiovascular medications: lessons learned and future directions. Prog Cardiovasc Dis. 2013;55(6):590-600.

15. Nagin DS, Odgers CL. Group-based trajectory modeling in clinical research. Annu Rev Clin Psychol. 2010;6:109-38.

16. Nagin DS. Group-Based Modeling of Development. Cambridge, MA: Harvard University Press; 2005.

17. Nagin DS. Group-based trajectory modeling: an overview. Ann Nutr Metab. 2014;65(2-3):205-10

18. Marcum ZA, Walker RL, Jones BL, et al. Patterns of antihypertensive and statin adherence prior to dementia: findings from the adult changes in thought study. BMC Geriatr. 2019;19(1):41.

19. Winn AN, Fergestrom NM, Neuner JM. Using group-based trajectory models and propensity score weighting to detect heterogeneous treatment effects: the case study of generic hormonal therapy for women with breast cancer. Med Care. 2019;57(1):85-93.

20. MacEwan JP, Silverstein AR, Shafrin J, Lakdawalla DN, Hatch A, Forma FM Medication adherence patterns among patients with multiple serious mental and physical illnesses. Adv Ther. 2018;35(5):671-85.

21. Feldman $\mathrm{CH}$, Collins J, Zhang Z, et al. Azathioprine and mycophenolate mofetil adherence patterns and predictors among Medicaid beneficiaries with systemic lupus erythematosus. Arthritis Care Res (Hoboken). 2019;71(11):1419-24

22. Franklin JM, Gopalakrishnan C, Krumme AA, et al. The relative benefits of claims and electronic health record data for predicting medication adherence trajectory. Am Heart J. 2018;197:153-62.

23. Hargrove JL, Pate V, Casteel CH, et al. Antihypertensive adherence trajectories among older adults in the first year after initiation of therapy. Am J Hypertens. 2017;30(10):1015-23.
24. MacEwan JP, Forma FM, Shafrin J, Hatch A, Lakdawalla DN, Lindenmayer JP. Patterns of adherence to oral atypical antipsychotics among patients diagnosed with schizophrenia. J Manag Care Spec Pharm. 2016;22(11):1349-61. Available at: https://www.jmcp.org/doi/10.18553/ jmcp.2016.22.11.1349.

25. Lo-Ciganic WH, Donohue JM, Jones BL, et al. Trajectories of diabetes medication adherence and hospitalization risk: a retrospective cohort study in a large state Medicaid program. J Gen Intern Med. 2016;31(9):1052-60.

26. Winn AN, Dusetzina SB. The association between trajectories of endocrine therapy adherence and mortality among women with breast cancer. Pharmacoepidemiol Drug Saf. 2016;25(8):953-59.

27. Franklin JM, Krumme AA, Shrank WH, Matlin OS, Brennan TA, Choudhry NK. Predicting adherence trajectory using initial patterns of medication filling. Am J Manag Care. 2015;21(9):e537-544.

28. Newman-Casey PA, Blachley T, Lee PP, Heisler M, Farris KB, Stein JD. Patterns of glaucoma medication adherence over four years of follow-up. Ophthalmology. 2015;122(10):2010-21.

29. Juarez DT, Williams AE, Chen C, et al. Factors affecting medication adherence trajectories for patients with heart failure. Am J Manag Care. 2015;21(3):e197-205.

30. Franklin JM, Krumme AA, Tong AY, et al. Association between trajectories of statin adherence and subsequent cardiovascular events. Pharmacoepidemiol Drug Saf. 2015;24(10):1105-13.

31. Wang CH, Huang LC, Yang CC, et al. Short- and long-term use of medication for psychological distress after the diagnosis of cancer. Support Care Cancer. 2017;25(3):757-68.

32. Hickson RP, Annis IE, Killeya-Jones LA, Fang G. Opening the black box of the group-based trajectory modeling process to analyze medication adherence patterns: an example using real-world statin adherence data. Pharmacoepidemiol Drug Saf. 2020;29(3):357-62

33. Vadhariya A, Fleming ML, Johnson ML, et al. Group-based trajectory models to identify sociodemographic and clinical predictors of adherence patterns to statin therapy among older adults. Am Health Drug Benefits. 2019;12(4):202-11.

34. Hernandez I, He M, Chen N, Brooks MM, Saba S, Gellad WF Trajectories of oral anticoagulation adherence among Medicare beneficiaries newly diagnosed with atrial fibrillation. J Am Heart Assoc. 2019;8(12):e011427.

35. Dillon P, Stewart D, Smith SM, Gallagher P, Cousins G. Group-based trajectory models: assessing adherence to antihypertensive medication in older adults in a community pharmacy setting. Clin Pharmacol Ther. 2018;103(6):1052-60

36. Dillon P, Smith SM, Gallagher P, Cousins G. The association between pharmacy refill-adherence metrics and healthcare utilisation: a prospective cohort study of older hypertensive adults. Int J Pharm Pract. 2019;27(5):459-67.

37. Aarnio E, Martikainen J, Winn AN, Huupponen R, Vahtera J, Korhonen MJ. Socioeconomic inequalities in statin adherence under universal coverage: does sex matter? Circ Cardiovasc Qual Outcomes. 2016;9(6):704-13.

38. Librero J, Sanfelix-Gimeno G, Peiro S. Medication adherence patterns after hospitalization for coronary heart disease. A population-based study using electronic records and group-based trajectory models. PLoS One. 2016;11(8):e0161381.

39. Märdby AC, Schioler L, Sundell KA, Bjerkeli P, Lesen E, Jonsson AK. Adherence to antidepressants among women and men described with trajectory models: a Swedish longitudinal study. Eur J Clin Pharmacol. 2016;72(11):1381-89.

40. Lambert-Cote L, Bouhnik AD, Bendiane MK, et al. Adherence trajectories of adjuvant endocrine therapy in the five years after its initiation among women with non-metastatic breast cancer: a cohort study using administrative databases. Breast Cancer Res Treat. 2020;180(3):777-90. 
41. Ajrouche A, Estellat C, De Rycke Y, Tubach F. Trajectories of adherence to low-dose aspirin treatment among the French population. J Cardiovasc Pharmacol Ther. 2020;25(1):37-46.

42. Chen CC, Cheng SH. Continuity of care and changes in medication adherence among patients with newly diagnosed diabetes. Am J Manag Care. 2016;22(2):136-42.

43. Schaffer AL, Buckley NA, Pearson SA. Who benefits from fixed-dose combinations? Two-year statin adherence trajectories in initiators of combined amlodipine/atorvastatin therapy. Pharmacoepidemiol Drug Saf. 2017;26(12):1465-73.

44. Tervonen HE, Daniels B, Tang M, Preen DB, Pearson SA. Patterns of endocrine therapy in a national cohort of early stage HER2-positive breast cancer patients. Pharmacoepidemiol Drug Saf. 2019;28(6):812-20.

45. Zongo A, Simpson S, Johnson JA, Eurich DT. Change in trajectories of adherence to lipid-lowering drugs following non-fatal acute coronary syndrome or stroke. J Am Heart Assoc. 2019;8(23):e013857.
46. Feldman CH, Collins J, Zhang Z, et al. Dynamic patterns and predictors of hydroxychloroquine nonadherence among Medicaid beneficiaries with systemic lupus erythematosus. Semin Arthritis Rheum. 2018;48(2):205-13.

47. Pharmacy Quality Alliance. PQA measure use in CMS' Part D quality programs. Available at: https://www.pqaalliance.org/medicare-part-d. Accessed July 8, 2020.

48. Centers for Medicare \& Medicaid Services. Medicare 2020 Part C \& D star ratings technical notes. October 1, 2019. Available at: https://www.cms. gov/Medicare/Prescription-Drug-Coverage/PrescriptionDrugCovGenIn/ Downloads/Star-Ratings-Technical-Notes-Oct-10-2019.pdf. Accessed July 8, 2020.

49. Abughosh SM, Vadhariya A, Johnson ML, et al. Enhancing statin adherence using a motivational interviewing intervention and past adherence trajectories in patients with suboptimal adherence. J Manag Care Spec Pharm. 2019;25(10):1053-62. Available at: https://www.jmcp.org/ doi/10.18553/jmcp.2019.25.10.1053. 